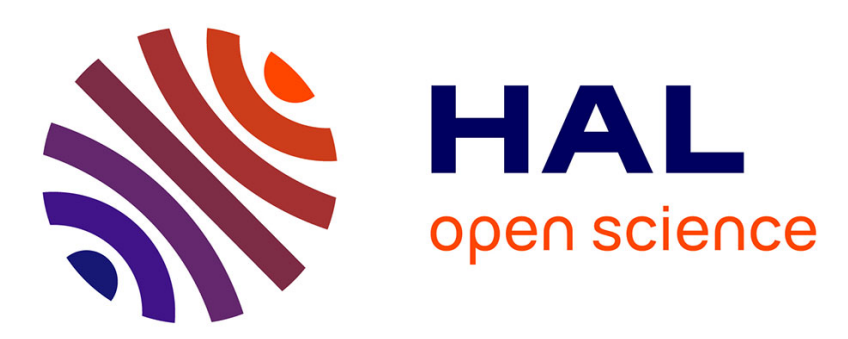

\title{
Les paysages lus du ciel. De l'intérêt de la photographie aérienne oblique
}

\author{
André Humbert, Roland Courtot, Colette Renard
}

\section{To cite this version:}

André Humbert, Roland Courtot, Colette Renard. Les paysages lus du ciel. De l'intérêt de la photographie aérienne oblique. Méditerranée: revue géographique des pays méditerranéens, 2013, Espaces agraires, dynamiques paysagères, structures foncières, acteurs et planification, 120, pp.111-126. 10.4000/mediterranee.6748 . hal-01556485

\section{HAL Id: hal-01556485 \\ https://hal.univ-lorraine.fr/hal-01556485}

Submitted on 5 Jul 2017

HAL is a multi-disciplinary open access archive for the deposit and dissemination of scientific research documents, whether they are published or not. The documents may come from teaching and research institutions in France or abroad, or from public or private research centers.
L'archive ouverte pluridisciplinaire HAL, est destinée au dépôt et à la diffusion de documents scientifiques de niveau recherche, publiés ou non, émanant des établissements d'enseignement et de recherche français ou étrangers, des laboratoires publics ou privés. 


\section{Méditerranée}

Revue géographique des pays méditerranéens / Journal of Mediterranean geography

\section{Les paysages lus du ciel. De l'intérêt de la} photographie aérienne oblique

Landscapes from the sky, the interest of aerial photography

\section{André Humbert, Roland Courtot et Colette Renard}

\section{revues.org}

Édition électronique

URL : http://mediterranee.revues.org/6748

ISSN : $1760-8538$

\section{Éditeur}

Presses Universitaires de Provence

Édition imprimée

Date de publication : 18 octobre 2013

Pagination : 111-126

ISBN : 978-2-85399-881-9

ISSN : 0025-8296

Référence électronique

André Humbert, Roland Courtot et Colette Renard, « Les paysages lus du ciel. De l'intérêt de la photographie aérienne oblique », Méditerranée [En ligne], 120 | 2013, mis en ligne le 30 mai 2015, consulté le 03 janvier 2017. URL : http://mediterranee.revues.org/6748 ; DOI : 10.4000/ mediterranee.6748

Ce document est un fac-similé de l'édition imprimée. 


\section{Les paysages lus du ciel}

\section{De l'intérêt de la photographie aérienne oblique}

\section{Landscapes from the sky, the interest of aerial photography}

\author{
André HUMBERT \\ Université de Lorraine, Loterr (ex-Cerpa) \\ andrehumbert@aol.com
}

Roland COURTOT

Université d'Aix-Marseille, Telemme

courtot@mmsh.univ-aix.fr
Colette RENARD

Université de Lorraine, Loterr (ex-Cerpa)
Résumé : La vision et la photographie aérienne ont enthousiasmé les géographes de la première moitié du $\mathrm{XX}^{\mathrm{e}} \mathrm{s}$., avant que l'intérêt pour ce type de document ne s'émousse avec de nouvelles orientations épistémologiques de la géographie. Les auteurs de cet article veulent montrer que la photographie aérienne oblique, grâce à l'éloignement indispensable qu'elle procure et à la lisibilité des paysages qu'elle offre, est un média d'une grande utilité pour l'analyse des espaces géographiques. Son manque de rigueur "géométrique " n'interdit pas que les résultats obtenus soient reportés sur des supports cartographiques à échelle constante ou sur des coupes dotées d'une double échelle, pour un rendu « horizontal » des informations recueillies. L'article est fondé sur deux études de cas méditerranéennes pour exposer une méthode qui a recours à la théorie des ensembles et une démarche « descendante » qui conduit des grands faciès des paysages aux objets géographiques significatifs.

Mots-clés : photographie aérienne oblique, analyse de l'espace géographique, croquis d'interprétation, faciès paysagers, paysages méditerranéens, épistémologie

Début de l'aviation et photographie aérienne sont concomitants. Dès l'aube du XX ${ }^{\mathrm{e}}$ siècle (1909-1910), l'armée française se dotait d'un nouvel outil d'investigation et de surveillance à usage interne, notamment pour la zone des fronts pendant la Grande Guerre (DUMARCHE, 1988) mais aussi à destination des géographes. Dans un article des Annales de Géographie (1924), E. de Martonne signale que le Comité national de Géographie, présidé par le général Bourgeois, a obtenu que l'aviation militaire prête son concours pour la prise de vues géographiques choisies par une commission de professeurs. C'est en 1947 que l'IGN reçut la mission de couvrir la totalité de la France par un survol systématique, standardisé et régulier de l'espace français mettant à disposition de nouveaux utilisateurs initiés à la photo-interprétation des milliers de clichés d'abord en noir et blanc, puis en couleurs. Depuis les années 1970, l'observation aérienne a été enrichie par l'apport des images satellitales très utilisées en télédétection. Aujourd'hui, la vue aérienne verticale s'est largement démocratisée en particulier par l'ouverture sur la toile de sites tels que ceux de la NASA, de Google Earth ou encore de Géoportail. Les photographies aériennes obliques sont, elles aussi, devenues très familières par le biais traditionnel des cartes postales, par celui de nombreux documents des manuels scolaires de géographie et, bien sûr, par celui de productions de très beaux ouvrages tels ceux d'A. MacLean (2008) et plus modestement d'H. Colson (2010), de courts ou longs métrages réalisés par des professionnels de la photographie, souvent des artistes (Y.-A. Bertrand). Les entreprises de photographies aériennes sont nombreuses et la vue aérienne oblique se vend bien! Paradoxalement si le cliché aérien oblique est prisé par les archéologues, par les entreprises, les collectivités territoriales et par les particuliers en tant que média de mise en valeur d'un patrimoine, il apparaît
Abstract: The vision and aerial photography have filled geographers with enthusiasm during the first half of the XXth century before the interest for this sort of document became tempered with new epistemological orientations of geography. the writers of this article want to show that the oblique aerial photography thanks to the essential distance it provokes and thanks to the readability of landscapes it affords is a media of great use to analyze geographical spaces. Its lack of "geometrical"rigour does not prevent reporting the results obtained on mapping imagery with a constant scale or on sections provided with a double scale to obtain a"horizontal"result of collected informations. The article is based on two studies of Mediterranean cases to put on display a method resorting to the set theory and a top-down approach which leads from large facies of landscapes to significant geographic objects.

Keywords: oblique aerial photography, analyze geographical spaces, facies of landscapes, Mediterranean landscapes, epistemology

moins plébiscité par la communauté des géographes qui lui préfèrent les orthophotos. La photographie aérienne oblique ne peut-elle pas être un objet d'analyse en tant que telle? En quoi la production de photographies aériennes obliques et leur interprétation participent-elles de l'analyse de l'organisation de l'espace?

Après une rétrospective non exhaustive de l'utilisation de la photographie aérienne par les géographes et une présentation des conditions de la production de clichés aériens géographiques, deux études de cas méditerranéens permettront de présenter des approches méthodologiques complémentaires d'interprétation.

\section{I - La photographie aérienne oblique: un outil assez peu utilisé par les géographes}

Avec le retour de la paix, les années 1920 furent assez riches en publications d'ouvrages originaux rassemblant des photographies aériennes verticales et obliques et d'articles soulignant l'avantage de la vue aérienne. Dans l'introduction de The face of the Earth as seen from the air, a study in the application of airplane photography to geography édité luxueusement par la Société de géographie de New York, en 1920, W. T. Lee note l'avantage des vues obliques qui sont, en fait, au nombre de trois: présenter des objets d'intérêt « populaire ", comme le Capitole, destinés à un grand public, aider à l'étude des « formes de terrain » et apporter une aide à la cartographie. Dans un compte rendu de lecture de l'ouvrage de O. Flückiger (1922) sur la Suisse, E. de Martonne (1924) souligne que le photographe-aviateur « doit être géographe » en raison du « coup d'œil qui tient du prodige ». Il invitait à la réalisation d'un travail analogue pour la France et concluait par ces mots : 


\begin{abstract}
La photographie aérienne est appelée à jouer un rôle de plus en plus grand dans la documentation géographique. Est-ce à dire que ce rôle devienne exclusif? Je ne le crois pas. La photographie ordinaire reste seule capable de rendre les détails. Elle seule représente toujours la terre telle que la voient les hommes. L'avion apporte un complément à la documentation photographique, complément précieux et qu'on peut dès à présent considérer comme indispensable.
\end{abstract}

D'autres travaux étaient en gestation avant le second conflit mondial mais n'ont été édités qu'à la fin des années 1940. C'est encore à E. de Martonne que nous devons les écrits les plus conséquents. À l'issue du premier congrès de géographie aérienne organisé par l'Union syndicale des Industries aéronautiques à la fin de 1938, il entreprit un précis de géographie aérienne publié dix ans plus tard. La troisième des quatre parties de cet ouvrage est intitulée « Physiographie aérienne ». L'auteur est de toute évidence conquis, enchanté. Pour lui l'avantage essentiel de la vision aérienne est de permettre à la fois la perception des détails et celle des ensembles, l'analyse et la synthèse. Mais il regrette la rapidité avec laquelle défilent les images. Si la photographie palie l'inconvénient du défilé trop rapide, la vue verticale prise sous un angle si différent de celui sous lequel nous sommes habitués à voir un paysage, engendre quelques difficultés à les interpréter. La photographie aérienne oblique apparaît moins déroutante. On ne s'étonnera pas que ce géographe affirme que c'est à l'étude des formes du relief que l'usage de l'avion semble apporter la contribution la plus importante.

Les nombreux volumes de la Géographie universelle dirigée par Paul Vidal de la Blache et Lucien Gallois utilisent encore peu les photographies aériennes. À titre d'exemple, dans l'ensemble du corpus photographique (133 clichés) du premier volume consacré principalement à l'économie agricole, aux grandes régions agricoles et à la vie rurale de la France économique et humaine, paru en 1946, Albert Demangeon a sélectionné 25 vues aériennes obliques (soit environ près de $20 \%$ ) et deux subverticales. Les clichés en noir et blanc réalisés pour le plus grand nombre par la Compagnie aérienne française ' , pionnière du travail aérien, sont regroupés par planches et ne sont accompagnées, le plus souvent, que d'un titre informatif du type « cultures irriguées aux environs d'Aigues-Mortes (Hérault)» (p. 73), parfois enrichi par un bref complément tel que:

Vue aérienne de Vitry-le-François

Ville forteresse au plan géométrique, fondée par François I ${ }^{e r}$ pour recueillir les survivants de Vitry-en-Perthois, rasée par Charles-Quint en 1544, (p. 254).

Les clichés sont toutefois appelés dans le texte de l'auteur, mais parfois beaucoup plus loin.
En revanche, deux collections de la deuxième moitié $\mathrm{du} \mathrm{XX}^{\mathrm{e}}$ siècle leur consacrent, au contraire, une place importante. C'est tout d'abord l'Atlas aérien ${ }^{2}$ de Pierre DEFFONTAINES et Mariel JEAN-BRUNHES DELAMARRE dont les volumes ont été publiés progressivement entre 1955 et 1964. Chacun des cinq tomes rassemble de 200 à plus de 300 clichés exclusivement en noir et blanc. À titre d'exemple, le tome I consacré aux Alpes, vallée du Rhône, Provence et à la Corse, rassemble 209 photographies dont 26 sont des photographies aériennes verticales réalisées par l'IGN, alors que toutes les autres sont des photographies obliques prises à très basse altitude telle la photo 145 « cultures d'oliviers sur la rive orientale de l'étang de Berre » ou au contraire, à une altitude beaucoup plus élevée comme la photo 165 de l'est du bassin de Bandol («Ollioules et ses jardins de primeurs et de fleurs » sur laquelle sont éparpillés des mas). Quelquefois sont juxtaposées photographie aérienne verticale et photographie aérienne oblique pour fournir, d'un même site, divers « éclairages » (photos 122-123, Caderousse en Comtat Venaissin). Chacune des photographies est accompagnée d'une « légende développée », c'est-à-dire d'un commentaire plus ou moins long, plus ou moins descriptif introduisant parfois des informations historiques expliquant le présent.

Cet ouvrage qui constitue une précieuse banque d'images datant du milieu du $\mathrm{XX}^{\mathrm{e}}$ siècle est introduit par un court texte intitulé " Nouvelles visions de la terre par avion » dans laquelle les deux auteurs évoquent la « révolution de vision » engendrée par l'avion, " le plus merveilleux mode de connaissance qui a remplacé la vision linéaire et à ras du sol par une vision de surface ou même en volume ». L'aéronef libère le voyageur terrestre enfoui dans les éléments du paysage et lui donne la vision totale qui permet de comprendre la composition d'un paysage, en faisant apparaître l'agencement des éléments. La vision aérienne est une « vision d'ensemble, de synthèse surtout ».

L'intérêt - la nécessité - de la distanciation a été compris par les géographes du début du siècle qui ont découvert avec émerveillement le spectacle du monde que leur offrait l'avion. Plus d'un demi-siècle plus tard alors que la plupart des sciences a découvert l'intérêt de la théorie des ensembles et de la systémique, le biologiste Joël de Rosnay affirme que les sciences n'ont pas seulement besoin d'outils offrant une grande acuité sur les objets étudiés, mais qu'il leur manque un macroscope leur donnant accès aux organisations ouvrant la voie à la compréhension des systèmes (DE ROSNAY, 1975). La géographie autant que d'autres sciences - sinon plus - a besoin de cet outil qui lui permet de s'élever au-dessus du corps des systèmes territoriaux pour commencer à comprendre leur logique ou, en tout cas, se poser des questions pertinentes sur leur agencement et leur fonctionnement. Nous disposons maintenant de ce macroscope qui nous permet de voir de très loin la « face de la terre ». Ces outils de prise de recul sont aujourd'hui nombreux mais la photo oblique

I Compagnie aérienne française, compagnie qui dès sa création en 1919 par Henri Balleyguier, polytechnicien et pilote, se spécialisa dans le travail aérien. Compagnie indépendante qui durant l'entre-deux-guerres réalisa des prises de vues jusqu'en Gaspésie et dans les colonies d'Afrique noire. Elle opéra jusqu'au lendemain de la Seconde Guerre mondiale.

2 Publications antérieures de photographies aériennes:

- Capitaine Seive et Cholley André, (1931), L’Atlas aérien du Rhône, Lyon, 54 planches (50 x 32,5 cm).

- Chombard de Lauwe P.H., (1948), Découverte aérienne du Monde, Paris, Horizons de France, 414 p., ouvrage préfacé par E. de Martonne. 
n'en est pas pour autant dépréciée. Elle donne accès aux organisations avec une grande souplesse d'utilisation. De plus, « l'observation par avion facilite [par la vitesse même du déplacement], les comparaisons, les oppositions ; elle incite à la réflexion. ».

D'autres publications ont aussi abondamment puisé dans les stocks de photographies aériennes obliques, qui seraient d'après Paul Veyret « les mieux adaptées à notre vision », pour illustrer d'autant plus richement que la couleur est introduite, des ouvrages de géographie régionale, parfois destinés au grand public. Ce fut le cas de la collection Découvrir la France, dirigée par Roger Brunet, dont les fascicules ou les sept volumes conçus par des universitaires ont été publiés entre 1972 et 1974. Le commentaire a disparu et il ne subsiste qu'un court texte (souvent moins de 100 caractères), concis localisant le paysage et soulignant une particularité paysagère. La photographie n'est plus matériaux, n'est plus analysée en tant que telle mais accompagne le texte principal et les cartes. Elle est au service d'un projet éditorial.

$\mathrm{Au}$ fil des tournants épistémologiques de la géographie, les ouvrages de géographie régionale se sont raréfiés en France. De ce fait, mais aussi de celui des coûts engendrés par la publication en quadrichromie, les publications géographiques utilisent moins la photographie aérienne oblique. Dans les ouvrages les plus récents, elle conserve essentiellement une fonction d'illustration parfois emblématique comme dans le cahier couleur du Dictionnaire de la mer et des côtes (MIOSSEC, 2012). Elles ne sont parfois que prétextes. La petite photographie aérienne de la station de Deauville vue de la mer reproduite en noir et blanc dans l'ouvrage de R. KNAFOU, Les lieux du voyage (2012), aurait mérité plus de considération et aurait pu être l'objet d'un croquis d'interprétation.

Les géographes espagnols, dans le cadre des communautés autonomes, semblent conserver davantage d'intérêt pour l'utilisation de ce type de documents comme en témoignent quelques ouvrages cités en bibliographie (BIELZA, 1994; MOLINERO, 2011).

La banalisation de la photographie aérienne a succédé à l'enthousiasme des premiers utilisateurs. La photographie aérienne verticale a supplanté la photographie aérienne oblique sans que les multiples avantages soulignés par nos prédécesseurs se soient estompés.

\section{2 - De la difficulté à se procurer les images souhaitées}

Un des reproches que l'on peut faire à la photographie oblique est qu'elle ne fournit pas aux géographes une couverture systématique, exhaustive, comme le font les photographies verticales de l'IGN. Les prises de vue obliques sont sinon aléatoires du moins réalisées en fonction d'une sélection qui relève des centres d'intérêt du géographe volant et aussi des circonstances du vol: celui-ci peut, bien entendu relever d'un projet d'observations sur une aire définie, mais il peut n'être qu'un vol de liaison au cours duquel est mis à profit le défilement des paysages sous les ailes de l'avion. Au cours d'un voyage entre Nancy et Agadir, ce sont des couloirs étroits, littoraux ou à l'intérieur des terres qui fournissent les collections les plus abondantes et les plus variées. Leur intérêt n'en est nullement diminué pour autant. Mais quels que soient les paysages observés et photographiés au cours d'un vol, le géographe pilote est soumis à une tension intense due à l'accumulation des tâches à la fois techniques et intellectuelles. L'architecte belge, Jean Dethier ${ }^{3}$, exprime bien cette charge dans l'interview qu'il accorde lors de la publication du magnifique ouvrage sur les paysages des États-Unis du pilote architecte américain MacLean (MACLEAN, 2008):

Le regard porté sur le monde avec la perspective de l'oiseau provoque une intense fascination, mais implique aussi de multiples difficultés dès lors qu'on veut assumer un travail utile et signifiant, qui dépasse la seule valeur esthétique ou anecdotique. En effet en vol, le regard est sollicité en toutes directions par une sorte de « spectacle total » $[. .$.$] qu'il s'avère très$ difficile d'assumer trois opérations nécessairement simultanées et complémentaires à celle du pilotage : [1] apprécier l'intérêt et la pertinence de ce que l'on peut voir tout autour de soi; [2] choisir dans l'immensité du paysage panoramique en mouvement le cadrage du détail approprié à photographier afin qu'il soit structurellement cohérent et visuellement plaisant; [3] prendre en compte les informations géographiques précises (lieu, orientation, etc. ) indispensables pour restituer scientifiquement le segment photographié dans le contexte de l'immensité du territoire parcouru. [...] Chacune de ses photographies est donc la résultante d'un extraordinaire et étonnant cumul de contraintes et d'exigences à assumer dans la fugacité de l'instant.

Des contraintes et des limitations sont aussi dues aux conditions du vol dit «à vue » qui est celui de la plupart des avions légers utilisés pour effectuer les photographies obliques. Si ce régime de vol présente l'avantage de laisser au pilote une grande liberté pour choisir ses itinéraires et pour effectuer des évolutions dans une zone d'intérêt, il faut cependant savoir qu'il est soumis à des contraintes très strictes dans certaines zones contrôlées par les militaires ou par les organismes de l'aviation civile qui gèrent des espaces souvent étendus autour des aéroports. Dans ces zones les avions sont soumis à des trajectoires qui ne sont pas toujours celles que souhaiterait le géographe. Pire encore, il est parfois rejeté hors de secteurs qui mériteraient d'être observés mais qui sont interdits, strictement, aux vols à vue en raison de contraintes particulières du trafic aérien. Un des meilleurs exemples est celui de la zone contrôlée qui entoure l'aéroport de Barcelone: environ $80 \mathrm{~km}$ de littoral, pourtant très intéressants pour le géographe, sont inaccessibles. D'autres interdictions sont moins strictes mais les possibilités de survol dépendent de la bonne volonté des

3 On lira avec le plus grand intérêt pour un géographe la conversation avec Jean Dethier, architecte et urbaniste belge résidant à Paris depuis 1970, située à la fin du livre d'Alex MacLean (2008), p. 326-331. 
contrôleurs, comme à Valence, en Espagne, où la trajectoire normale est imposée assez loin de la côte à travers le golfe. Un survol du littoral, entre le port de Valence et le rocher de Cullera, est du plus grand intérêt, mais au cours des nombreux transits que nous avons effectués, ce survol nous a été souvent refusé pour des raisons tout à fait valables de trafic autour de l'aéroport, ou tout simplement en raison de la mauvaise humeur du contrôleur. D'autres limitations proviennent de la réglementation concernant le survol des agglomérations de grande taille qui oblige les aéronefs à prendre de l'altitude et donc à perdre de l'acuité même si les objectifs à longue focale permettent de compenser partiellement ce handicap. Au Maroc, de surcroît, un nombre important de villes sont interdites de tout survol en raison de la présence en leur sein d'un palais royal. Chaque fois que l'on s'approche de l'aéroport d'une de ces villes, le contrôleur ne manque pas de rappeler cette interdiction de façon très énergique.

Il existe donc certaines étendues blanches dans la couverture aérienne en photographie oblique, ceci pour des raisons qui peuvent tenir à des choix délibérés des géographes volants mais aussi parce que règnent sur ces espaces des contraintes de nature réglementaires ou politiques.

En comparaison des prises de vues automatisées qui procurent aux utilisateurs une couverture intégrale de vastes espaces, la photographie aérienne oblique suppose donc l'acceptation par le géographe pilote de nombreuses contraintes qui lui laissent, néanmoins la possibilité de procéder à la sélection d'images choisies; celles-ci correspondant à une analyse « à chaud » du paysage et au découpage de scènes qui répondent à une problématique surgie de la perception d'un espace survolé.

\section{3 - Des paysages lus du ciel}

Si la photographie aérienne verticale a été et est très exploitée par les géographes, la photographie oblique est souvent réduite à une fonction d'illustration, dans le meilleur des cas accompagné d'un commentaire plus ou moins étoffé qui peut être d'ailleurs un texte relatif au lieu représenté sur le cliché sans qu'il s'agisse d'une analyse de l'image ou de l'espace photographié.

Peu de géographes se sont essayés à la lecture de ce type de clichés et à la production de représentations spatiales. On pourra s'étonner qu'en 1942, André Cholley dans le Guide de l'étudiant en Géographie n'y fasse aucune référence dans le chapitre qu'il consacre à la description géographique et même dans une sous partie intitulée « Description par l'image ». C'est plutôt vers les didacticiens ou vers les universitaires qui n'ont pas opéré de coupure entre la géographie universitaire et la géographie enseignée dans le secondaire qu'il faut se tourner. Quelques productions méritent d'être soulignées. Toutes proposent des croquis d'interprétation, certaines récusent le découpage en plans verticaux, d'autres composent avec ce découpage qui est strictement visuel. Ce fut tout d'abord le dossier produit par P. et G. PINCHEMEL (1987) dans lequel les auteurs subdivisent le paysage observé d'un point de vue panoramique ou aérien à relative basse altitude en unités paysagères. La démarche dépasse la «simple analyse paysagère »(LEGOUY, 2006) par l'intégration d'un grand nombre de données internes et externes pour expliquer le paysage. Pour F. Legouy, ce faisant, on s'écarte de l'analyse paysagère stricto sensu et imperceptiblement on passe à l'analyse spatiale. Unités paysagères correspondant aux vues du dedans et vues obliques et unités spatiales correspondant, elles, aux vues du dessus ou vues verticales sont différentes mais découlent d'une même réalité et constituent un couple que F. Legouy qualifie d'unité spatiopaysagère. Pour lui, une analyse complète paysagère et spatiale doit comporter deux lectures différentes, l'une objective et l'autre subjective qui ne se préoccupe que de la perception du paysage. Il décompose la première en quatre démarches distinctes :

- une analyse paysagère à partir de points de vue obliques combinant décomposition par plans verticaux pour tenir compte du glissement des échelles et unités paysagères;

- une analyse diachronique et dynamique du paysage à l'aide de documents photographiques anciens;

- une analyse spatiale qui distingue à partir des photographies aériennes et de la carte au 1/25000 des unités spatiales;

- et enfin une analyse environnementale qui consiste à dégager les rapports de l'homme à son milieu.

Un dossier conçu par J.-P. AMAT et C. GRATALOUP (1992) invite à travailler sur les modes de représentation du paysage, en deux temps. Après avoir identifié les objets élémentaires qui composent un paysage photographié (phase de démontage) et recherché « les agents et les processus, naturels et sociaux, qui organisent l'espace en ensembles visuels » est construite, à partir de ces mêmes objets élémentaires, une représentation graphique les regroupant en unités fonctionnelles, en tenant compte de leurs relations et de leurs interactions (phase de recomposition dans une logique systémique).

\section{I - Une méthode de lecture et d'interprétation des images aériennes obliques}

Nous allons exposer cette méthode d'analyse des images aériennes obliques à partir d'exemples choisis dans le domaine méditerranéen mais applicable dans n'importe quel contexte géographique.

Le principe de base est d'entrer toujours dans un paysage observé, par le haut et non par des détails, afin de dégager d'emblée les grandes articulations de l'agencement spatial d'une scène. Cette approche lointaine est largement intuitive: il s'agit seulement de mettre en évidence des différences manifestes dans la répartition des masses du paysage, de déceler des discontinuités, des ruptures qui séparent des ensembles sinon homogènes du moins suffisamment distincts les uns des autres pour qu'ils apparaissent comme des personnalités au faciès original.

Si ces grands faciès ne sont pas forcément homogènes dans leur contenu, cela signifie que, dans leur particularisme, ils peuvent, à leur tour contenir des sousensembles suffisamment différenciés pour être repérables. Ce n'est pas toujours le cas. Quoi qu'il en soit, la diversité 
à l'intérieur des faciès - ou des sous-ensembles - s'exprime à travers des agencements et des collections d'objets qui, par leur présence, expriment des fonctions actuelles ou passées.

Plus on avance dans la " profondeur » des paysages, dans leur intimité, plus l'observation devient précise, « raisonnée », plus elle demande de culture géographique, de connaissance des mécanismes fonctionnels et des formes qui leur sont associées. S'il n'est pas nécessaire d'être géographe pour distinguer des faciès dans un paysage, il faut avoir, en revanche, une culture géographique solide pour reconnaître un type d'habitation, une culture particulière (maïs, riz...), une usine chimique ou une cave coopérative.

Toutes les étapes - tous les niveaux - sont cependant solidaires; la vue distanciée présente l'avantage décisif de ne jamais séparer ces niveaux et de les mettre constamment en relation: un objet significatif - caractéristique est localisé dans un faciès - éventuellement dans un sous-ensemble de faciès -, donnant du sens à ces espaces.

Cette analyse «descendante » trouve son prolongement naturel dans une expression graphique qui résume les étapes de l'observation et fournit un outil didactique appréciable.

Le principe de cette représentation graphique est simple puisqu'il restitue, grâce à une différenciation claire des figurés, les grandes étapes de l'analyse.

Le premier niveau, celui des grands ensembles - ou des faciès - est doté d'une figuration uniquement composée d'à-plats colorés analogiques qui évoquent l'aspect général des faciès: les terroirs de culture pluviale sont souvent dans des teintes ocres ou brun clair, alors que les surfaces irriguées comme les rizières sont plutôt représentées en vert.

Le second niveau, quand il existe, est pourvu de figurés qui ne peuvent être confondus avec ceux du premier niveau; c'est pour cela que sont retenus des figurés linéaires ou ponctuels, en noir, qui se superposent facilement à la plage colorée des faciès sans l'occulter.

Les objets géographiques, enfin, sont représentés de façon diverse en fonction de leur nature et de leur étendue: ce peut être la silhouette d'un édifice quand celui-ci est facilement identifiable, mais parfois seulement le tracé du périmètre d'une superficie remarquable mais d'étendue limitée avec indication de sa nature; dans certains cas il suffit d'indiquer la localisation d'un objet, qui ne peut être confondu avec d'autres, par un symbole simple numéroté dont la signification est portée dans la légende.

Cette légende, précisément, doit obéir aux mêmes règles de présentation que le reste du document, c'est-à-dire que la hiérarchie spatiale doit être restituée par un artifice graphique qui montre l'emboîtement des divers niveaux spatiaux : la taille et la disposition des cartouches traduit le rang des espaces représentés et les liens fonctionnels sont traduits par les branche d'une arborescence. Grâce à cette légende un ordre est établi et les liaisons fonctionnelles sont exprimées clairement. L'espace - le territoire qui peut recouvrir tout un géosystème local - est expliqué dans son état le plus récent avec ses tendances évolutives quand des objets suffisamment significatifs le permettent. La comparaison avec d'autres documents favorise même l'établissement d'un schéma d'évolution rétrospective.

\section{2 - Une image relativement simple d'un paysage provençal}

Cet exemple offre une scène relativement simple et bien contrastée située au sud de l'extrémité occidentale des Alpilles, incluant la bordure de la Crau, à l'est d'Arles (fig. 1, 2 et 3$)$.

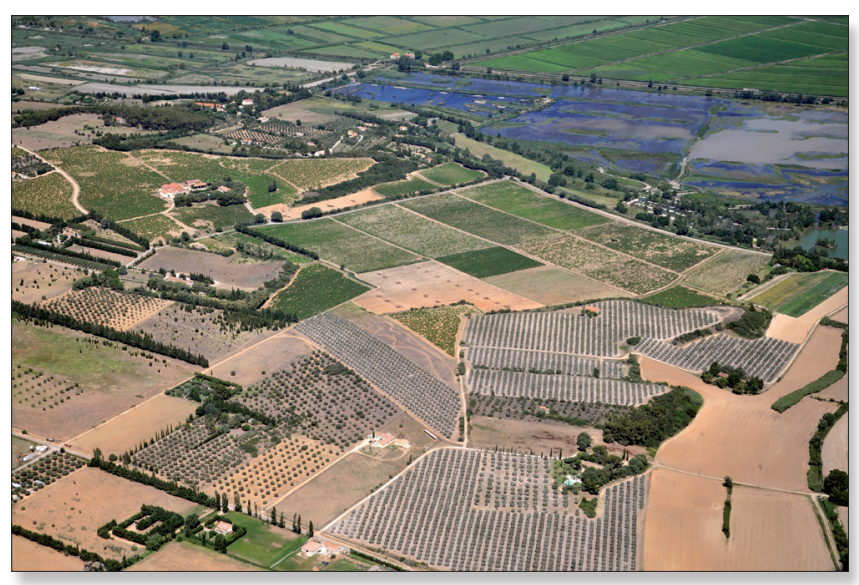

Fig. 1 - La Crau et la Vallée des Baux. Cliché A. Humbert et C. Renard, 21/07/2012.

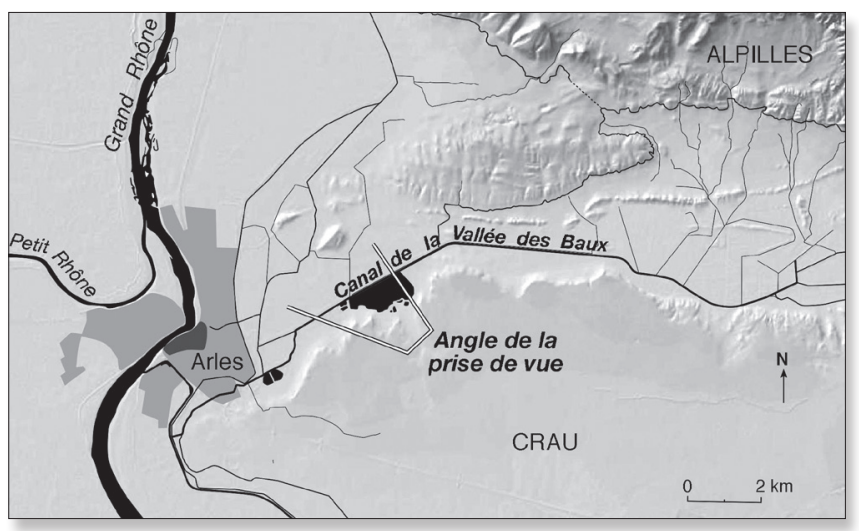

Fig. 2 - Carte de localisation du cliché de la figure 1. A. Humbert.

\subsection{I - Les grands faciès}

Trois s'imposent presque spontanément.

- Un ensemble de tonalité ocre dominante qui contraste de façon radicale avec le reste de la photo, ne serait-ce que par la teinte générale et le type de compartimentage spatial

Le reste du document est divisé en deux parties séparées par une ligne rigoureusement droite:

- En avant de cette ligne, la bande axiale du document apparaît comme un ensemble envahi par l'eau ou formant, du moins, une zone humide dans laquelle on discerne, cependant, un compartimentage plus ou moins évident.

- Le faciès le plus éloigné est marqué à la fois par une tonalité verte dominante et par un grand systématisme du découpage spatial.

\subsection{2 - Des sous-ensembles}

Si des différences tranchées ont permis de distinguer trois faciès majeurs; on constate facilement qu'un de ces faciès - le premier - présente en son sein deux sous-ensembles assez contrastés même s'ils conservent la tonalité générale du faciès.

- Le premier tire son individualité de la présence dominante de surfaces arborées régulièrement plantées. 


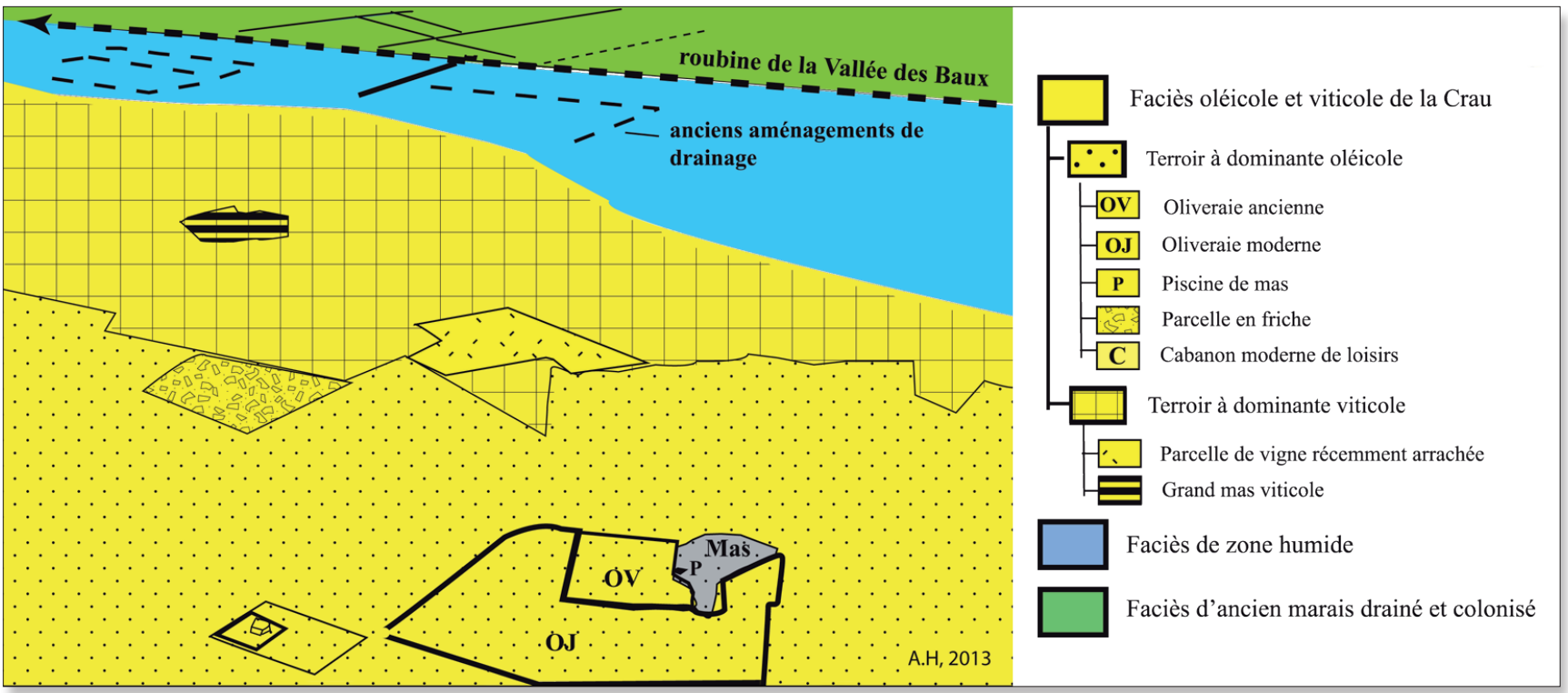

Fig. 3 - Croquis d'interprétation de la photographie de la figure 1. A. Humbert.

Une familiarité, même superficielle, avec les paysages méditerranéens permet de reconnaître des oliveraies;

- Le second sous-ensemble est dominé aussi, largement, par une culture un peu plus difficile à identifier mais dont la teinte et les alignements plus serrés, mais néanmoins bien distincts, mènent l'observateur à reconnaître de la vigne.

Il est donc légitime de diviser le premier faciès en deux sous-ensembles pourvu d'une certaine cohérence qui n'exclut pas les nuances internes. Si le premier est dominé par la présence des oliviers, il contient néanmoins des éléments qui ne sont pas oléicoles mais qui ne remettent pas en cause sa caractéristique majeure. Ces éléments étrangers à la dominante ne sont pas sans signification et ils interviendront dans la troisième étape de l'analyse. La même remarque peut être faite à propos de l'autre sousensemble dominé par la vigne qui comporte, par exemple, quelques parcelles d'oliviers.

\subsection{3 - Les objets géographiques marqueurs de fonction des territoires}

La physionomie des faciès - ou des sous-ensembles est le résultat de la combinaison, sur le terrain, d'un nombre variable, mais souvent important, d'objets - ou de collection d'objets - d'origine naturelle ou anthropique. Ces associations - ces mélanges - vus de haut, donnent leur caractère au tableau d'ensemble d'abord perçu et ressenti plutôt que compris. Ce sont ces objets, linéaires, ponctuels, aux formes complexes souvent, qui nous donnent le sésame - parfois incomplet - de l'organisation fonctionnelle de l'espace observé. Tous sont chargés d'information mais le choix des marqueurs les plus significatifs suppose une solide culture géographique non seulement pour leur identification mais également - et surtout - pour le choix des éléments les plus pertinents, choix capable de conduire à la compréhension du fonctionnement et de la genèse d'un territoire. Dans l'exemple proposé, les objets les plus caractéristiques sont évidemment les parcelles d'oliviers ou de vigne, mais un œil un tant soit peu exercé saisit des nuances parfois subtiles entre un champ de vieux oliviers et une plantation moderne, plus dense et aux alignements plus rigoureux. La ferme isolée au milieu de ses pièces de terre - le mas - est évidemment un élément important concernant l'organisation de l'habitat et les structures agraires, mais la présence - facilement repérable d'avion de piscines auprès des bâtiments de ferme trahit l'évolution de certaines exploitations vers des formes d'habitat résidentiels. Dans certains cas, la relation entre l'habitation et l'exploitation de la terre est même devenue incertaine ou tout à fait improbable.

L'image aérienne envoie des signaux clairs au géographe, mais certaines interprétations d'objets ou de relations sont douteuses parce que la distance est trop grande ou simplement parce que le phénomène - ou l'objet - n'est pas connu de l'observateur. Il suffit parfois de s'approcher davantage mais il peut être nécessaire d'avoir recours à d'autres sources d'information.

Celles-ci sont nombreuses et elles varient en fonction de la nature du questionnement. Les informations les plus variées, et souvent les plus sûres, sont celles que l'on recueille sur le terrain de la part des acteurs, de ceux qui ont planté la nouvelle oliveraie ou de ceux qui ont arraché la parcelle de vigne, avec les motivations et les conditions économiques associées à ces actes. Les entretiens de terrains peuvent aussi contribuer à éclairer la dynamique des espaces, sur le chemin d'une genèse plus ou moins complexe et plus ou moins « profonde».

La mémoire humaine se révèle rapidement insuffisante et fragile, mais d'autres sources peuvent prendre le relais : publications anciennes, rapports techniques, cartes à grande échelle établies à une époque éloignées du présent. Parmi ces documents historiques riches d'informations comparatives figurent, pour la région qui nous intéresse, les cartes d'état-major dressées au 1/40 000, dans la seconde moitié du XIX ${ }^{e}$ s. L'examen de la portion de carte correspondant à notre scène photographique est très instructif. Nous observons que le faciès correspondant 
au rebord de la Crau (au premier plan) est déjà un espace oléicole; il l'est même de façon plus exclusive qu'aujourd'hui car l'olivier occupe toute la surface qui est actuellement le domaine de la vigne; les arbres sont, cependant, parfois cantonnés sur les bordures des pièces de terre.

Cette carte est plus intéressante même pour les autres faciès qui sont encore représentés comme totalement marécageux ou lagunaires (étang de Peluque ou du Grand Char, étang du Petit Char) mais sont parcourus par un long et rectiligne canal de drainage, la roubine de la Fauque, c'està-dire le canal de la vallée des Baux des cartes actuelles. Alors que le dessin parcellaire est bien mis en évidence sur les autres parties de la carte grâce aux alignements d'arbres de bordure ou grâce au réseau de canaux d'irrigation dérivés du canal de Craponne, un peu plus au sud, et hors du champ de la photographie, le compartimentage actuel, parfaitement lisible sur la photo, est totalement inexistant à l'époque ; seules sont dessinées quelques roubines secondaires qui rejoignent les étangs ou le grand canal de drainage. La comparaison des deux documents permet de dater - de façon très relative - l'aménagement des zones humides de la vallée des Baux. Des documents intermédiaires permettraient d'affiner cette datation.

\section{3 - Un paysage complexe du Levant espagnol: la plaine littorale de Pego-Oliva}

\subsection{I - L'image d'un transect}

Le survol du littoral méditerranéen espagnol offre une riche palette de paysages qui se prêtent particulièrement bien à une interprétation d'espaces fonctionnels qui connaissent, ou ont connu, des transformations profondes.

L'image choisie pour l'analyse présente un transect de la plaine littorale plus ou moins dilatée qui prolonge, vers le sud, la Huerta de Valence (fig. 4).

Le cliché est pris depuis la mer jusqu'à l'alignement montagneux qui forme une sorte de toile de fond. Elle correspond, en gros, au territoire de la commune de Pego (province d'Alicante), même si celui du municipe voisin de Oliva (province de Valence), déborde en partie, dans la partie inférieure de l'image choisie. Le paysage est certes complexe mais il se décompose facilement en grands faciès dont les limites sont souvent très rectilignes et parallèles au front de mer. Cet exemple plus riche que le précédent nous donnera l'occasion de préciser un peu la méthode de décomposition en niveaux d'organisation, en exprimant graphiquement la progression de l'analyse spatiale. Cette image très didactique par la simplicité relative du découpage de l'espace nous apporte une richesse foisonnante d'informations sur un espace qui a connu naguère et récemment des transformations très radicales.

La simplicité du découpage global est soulignée par un certain nombre de lignes de forces parallèles au rivage mais surtout par des teintes très tranchées entre les différents compartiments ainsi que dans des contrastes de texture évidents. Dans un premier temps, il est facile de distinguer trois grandes bandes qui s'opposent fortement par leur occupation ou leur relief. Le premier plan du cliché a été mis volontairement en évidence car c'est cette bande littorale qui présente l'anthropisation la plus dense et la plus complexe. Le second ensemble, qui couvre presque la moitié du cliché est à l'évidence un faciès rural dont l'hétérogénéité pourra être analysée dans une seconde étape. Enfin, dressé au-dessus de la plaine, le relief montagneux pré-littoral est appréhendé plus comme une toile de fond que comme un faciès fonctionnel, en raison de son éloignement visuel. Ces trois ensembles - les grands faciès - constituent la première étape de l'analyse spatiale du paysage aérien. Nous pouvons exprimer graphiquement ce découpage par trois figurés en à-plats colorés comme nous l'avons fait pour le paysage provençal de la Crau. Le résultat est le croquis très simple de la figure $5 \mathrm{~A}$ accompagné de sa légende. Dans l'étape suivante nous affinons l'observation afin de dégager des sous-ensembles cohérents. Le faciès littoral n'est sans doute pas le plus facile à disséquer et, à ce stade il faut une bonne connaissance de l'organisation d'un complexe touristique pour distinguer l'ensemble balnéaire construit fait d'hôtels et d'immeubles d'appartements d'un sous-ensemble organisé autour d'un terrain de golf facilement reconnaissable à ses bunkers ensablés et à son resort qui a l'aspect d'un pseudo-village. La plage avec ses installations mobiles et les quelques dizaines de mètres de mer peuvent être considérés comme le sous-ensemble sand and sea du complexe touristique.

Le grand faciès que nous avons qualifié de rural est sans doute le plus facile à disséquer car les bandes parallèles au rivage se distinguent sans ambiguïté les unes des autres à la fois par leur teinte et leur texture. La plus éloignée mais aussi la plus importante est d'un vert soutenu très uniforme; elle est trop distante pour que l'on puisse lire clairement la texture du parcellaire, mais sa forme générale en éventail disposé sur ce qui est, à l'évidence un cône de déjection très aplati, et la perception de l'orientation des champs dans la partie inférieure indiquent sans doute des agencements parcellaires conformes à la pente générale du terrain. Une connaissance même sommaire de ces paysages méditerranéens conduit à identifier cette masse compacte comme une quasi monoculture d'agrumes. Le sous-ensemble le plus proche présente un aspect bien différent avec sa mosaïque parcellaire hétérogène et une variété culturale dans laquelle on remarque surtout les vergers d'agrumes. Si l'ambiance culturale est la même au-delà de la coupure nette que dessine le trait rectiligne de l'autoroute, on observe cependant une structure parcellaire différente avec une série de parcelles allongées et parallèles. Cette différence est source de questionnement mais elle ne remet pas en cause la tonalité générale du sous-ensemble ni son rôle fonctionnel.

Entre les deux sous-ensembles agricoles qui viennent d'être évoqués, il y a cette bande que l'on peut qualifier immédiatement d'humide. En effet, l'eau est présente dans la partie axiale de ce qui apparaît comme une gouttière littorale emprisonnée entre un cordon bien égoutté et un piémont en pente douce. Le caractère amphibie du reste n'est guère douteux car, si l'on observe attentivement la surface brune uniforme qui ne correspond à aucune culture connue, on s'aperçoit que celle-ci est trouée de "mares » plus ou moins étendues et plus ou moins régulières. Il est vrai, cependant que la bordure occidentale de cette zone humide, 


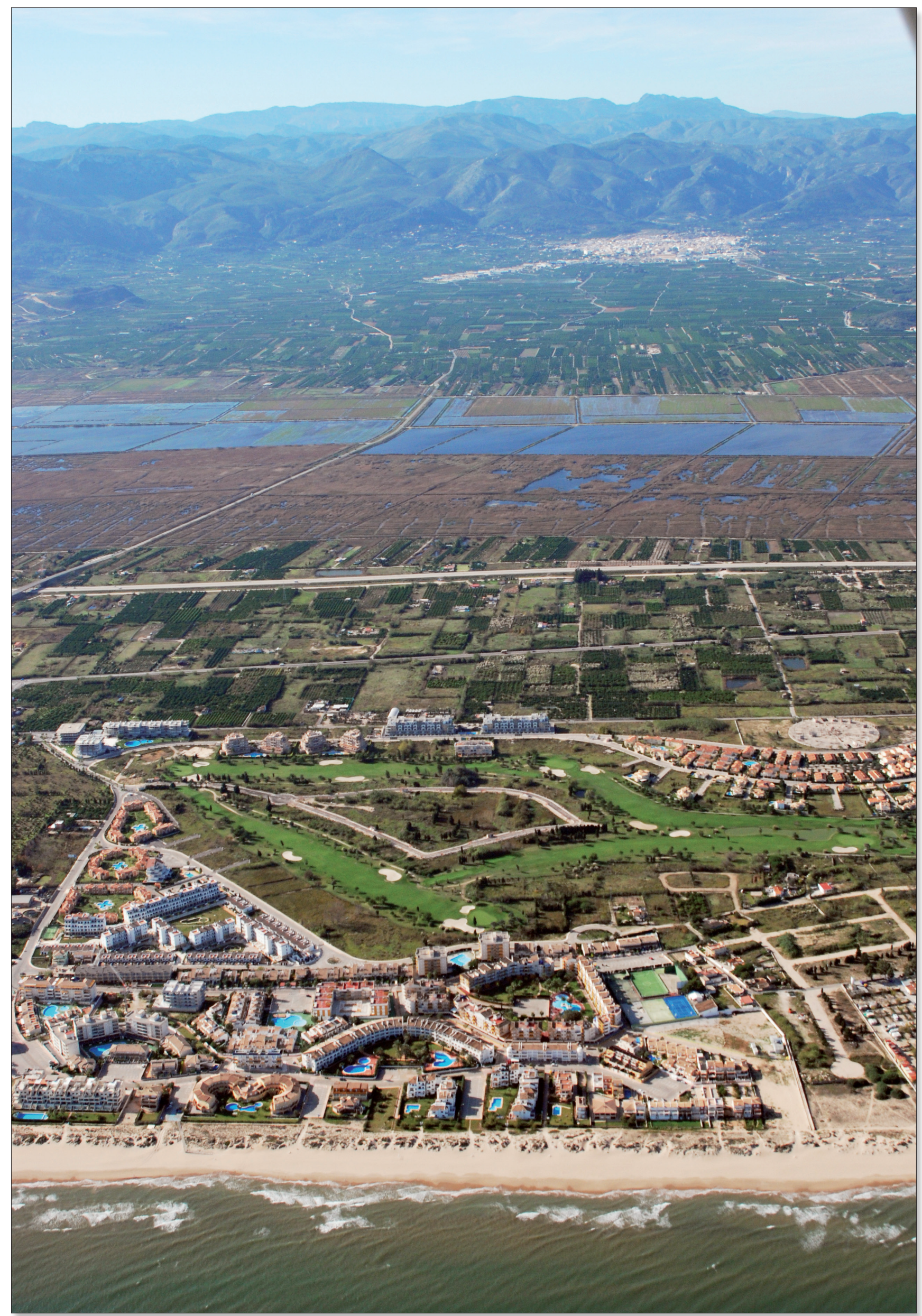

Fig. 4 - La plaine littorale de Pego vue vers le sud-ouest. Cliché A. Humbert, 4/11/2009. 


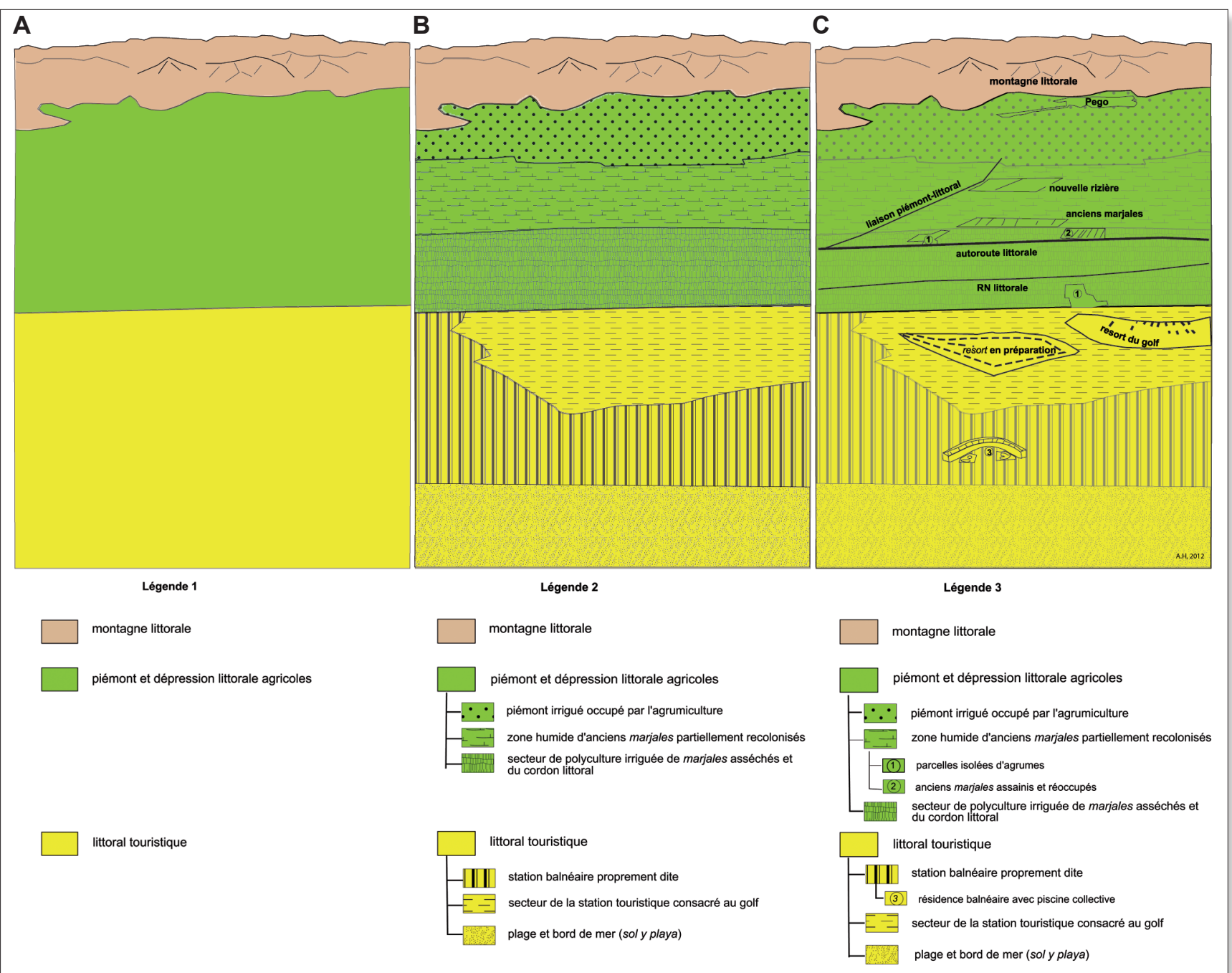

Fig. 5 - Croquis d'interprétation de la photographie de la figure 4. A: faciès, B: faciès et sous-ensembles, C: faciès, sous-ensembles et objets géographiques. A. Humbert.

tout en restant marquée par la présence de l'eau, présente une organisation parcellaire évidente. Cela est, à nouveau, source de questionnement. Le croquis d'interprétation rend compte de cette diversité interne des faciès grâce à la famille de figurés attribuée à ce niveau. Le croquis, exprime l'emboîtement des espaces.

Le troisième niveau de l'analyse spatiale est celui des objets, des objets géographiques, c'est-à-dire, parmi les innombrables items qui, rassemblés, mêlés forment la toile des paysages, ceux auxquels il est possible d'attribuer un rôle fonctionnel évident et majeur (fig. 5C).

Pour le faciès touristique, ce sont quelques formes caractéristiques d'hébergement, une piscine, le golf, bien évidemment, témoin d'une diversification de l'offre qui est faite. Pour les sous-ensembles de la partie agraire, c'est, bien entendu le gros village, centre d'un géosystème que l'on peut envisager s'étalant de la montagne au cordon littoral en grande partie confisqué aujourd'hui pour d'autres usages. Ce sont aussi les parcelles d'agrumes de la mosaïque du bas cordon littoral ainsi que quelques champs à l'organisation plus systématique qui correspondent à une conquête récente sur la zone humide. La gouttière humide mérite aussi que soient retenues quelques formes, tels que les grands rectangles en eau, en automne, qui signalent un usage particulier de la dépression. Ces grandes parcelles sont des casiers de rizières tels que ceux que l'on observe plus au nord dans la région de Cullera ou de la Albufera de Valence. Une brève histoire de la commune de Pego nous enseigne que la culture du riz a toujours été présente dans la gouttière pré-littorale depuis au moins le XVIII ${ }^{\mathrm{e}} \mathrm{s}$, avec un maximum de 900 ha après le guerre civile, en 1945, cette « poussée » céréalière correspondant à la politique frumentaire appliquée par le régime pour assurer une nourriture de base à une population affamée. Mais nous verrons, grâce à d'autres vues centrées sur la zone humide que des formes différentes, vraisemblablement plus anciennes ont permis de coloniser les lagunes (albuferas) pour une culture plus diversifiée. Sur cette vue, l'humedal n'est qu'en partie occupé par les cultures : un large secteur est abandonné à une végétation aquatique.

Cette vue statique du littoral levantin nous a apporté une quantité appréciable d'informations mais cette collecte peut être améliorée par des vues complémentaires qui peuvent ouvrir le panorama ou, au contraire, focaliser l'attention sur des détails révélés par un rapprochement ou un changement de l'angle de prise de vue. À titre d'exemple, nous avons sélectionné trois clichés qui complètent utilement le document principal.

\subsection{2 - Des regards complémentaires}

Le simple fait de pivoter de $90^{\circ}$ offre un regard totalement différent et ouvre une perspective sur un long segment de 


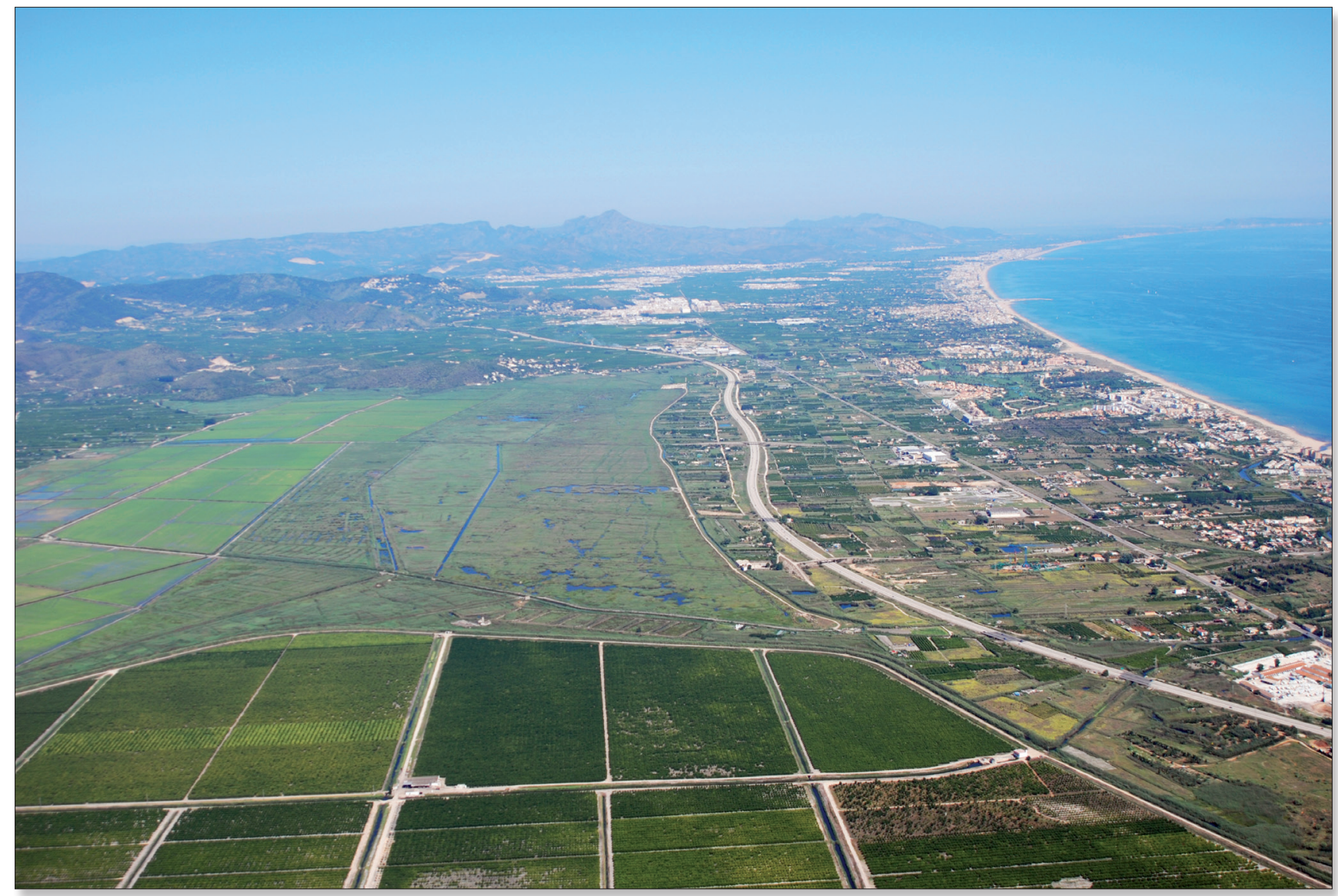

Fig. 6 - Le littoral vers le nord depuis Pego-Oliva. Cl. A. Humbert, 22/06/2008.

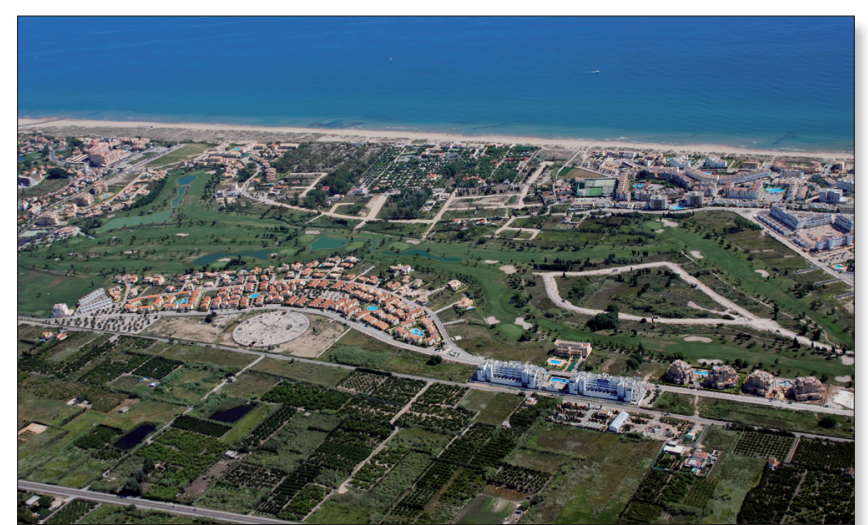

Fig. 7 - La « plage » de Pego-Oliva vue vers la mer. A. Humbert, 22/06/2008.

littoral qui s'étend jusqu'à l'entrée de la Huerta de Valence proprement dite. Sous cet angle, l'urbanisation intense observée sur un bref tronçon, se manifeste tout au long de la côte presque sans solution de continuité mais des nuances se font jour pour ce qui concerne l'occupation de la plaine qui, parfois, se dilate et accueille un archipel dense de villages et même la ville de Gandía, noyés dans le vert sombre des orangeraies qui s'avancent jusqu'au cordon littoral.

Cette orientation du cliché permet aussi une meilleure analyse de certains faciès et tout particulièrement celui des zones humides des anciens marjales abandonnés ou convertis en rizières et jeunes orangeraies. Enfin, cette vue méridienne souligne la prééminence des liaisons parallèles à la côte en mettant en évidence les grandes voies de communications (ici autoroute et chemin de fer) reliant le géosystème local, non seulement aux systèmes littoraux voisins, mais surtout aux aires continentales lointaines consommatrices de fruits et légumes méditerranéens et pourvoyeuses de cohortes amatrices de soleil et de plage.

Cette vue partielle (fig. 7) de la scène principale apporte quelques compléments intéressants, principalement, sur le cordon touristique proprement dit, en offrant l'intégralité du domaine consacré au golf avec son resort et ses extensions foudroyées par l'éclatement de la bulle immobilière. Le léger décalage de la visée vers le nord met en évidence un phénomène d'occupation touristique qui n'apparaît pas sur la vue principale: le vaste terrain de camping-caravanning, les «pieds dans l'eau » qui exprime l'importance de ce mode d'hébergement ancien et ubiquiste au long du littoral espagnol. Le fragment de terroir du premier plan est offert, sous cet angle, avec plus de précision: quelques mares résiduelles confirment l'ancienne appartenance du secteur aux marjales, les orangers apparaissent bien comme la culture dominante actuelle mais de nombreuses parcelles sont en friche - vraisemblablement des friches sociales - au contact avec le front d'urbanisation touristique.

Une vue rapprochée sur une partie de l'ample gouttière humide entre le cordon littoral et les terres bien égouttées du piémont agrumicole, résume parfaitement l'évolution d'un domaine littoral qui avait été colonisé dès le Moyen Âge par le biais du système des marjales. De façon on ne peut plus didactique, l'image montre comment une partie importante du domaine est aujourd'hui occupée par le parcellaire fossile des étroites parcelles séparées de fossés de l'ancien terroir paysan. Dans l'angle inférieur gauche du cliché, de nouvelles rizières aux grandes parcelles géométriques sont 
une forme de conversion du milieu humide qui ne renonce pas à l'environnement aquatique. Enfin, sur la droite, le choix a été fait de bonifier plus complètement l'ancien marais pour le coloniser par des orangeraies, sur une surface endiguée et drainée (tancat), la culture reine de la région, qui traverse aujourd'hui une grave crise après avoir souffert d'un manque de terres disponibles.

\section{4 - Les deux basculements possibles de l'image oblique - Changements de points de vue et modélisation graphique}

\section{I - La vision verticale: orthophotographie et carte}

L'étape suivante consiste donc à chercher sur la carte et la photographie aérienne en projection orthogonale, l'extension en plan des zones définies dans la partition précédente: les limites sont tracées à partir des images prises à la verticale, dans la mesure où elles fournissent suffisamment d'informations pour reconnaître les mêmes « homogénéités ». Une cartographie schématique se dessine alors sur une « région " plus vaste. Les nouveaux sites internet d'images de la terre sont alors un outil efficace (Géoportail, Iberpix Google earth, Nasa wordwind, Bing maps, etc.)

La partition définie dans l'espace de la photographie aérienne oblique (fig. 4 et 5) est projetée sur la carte

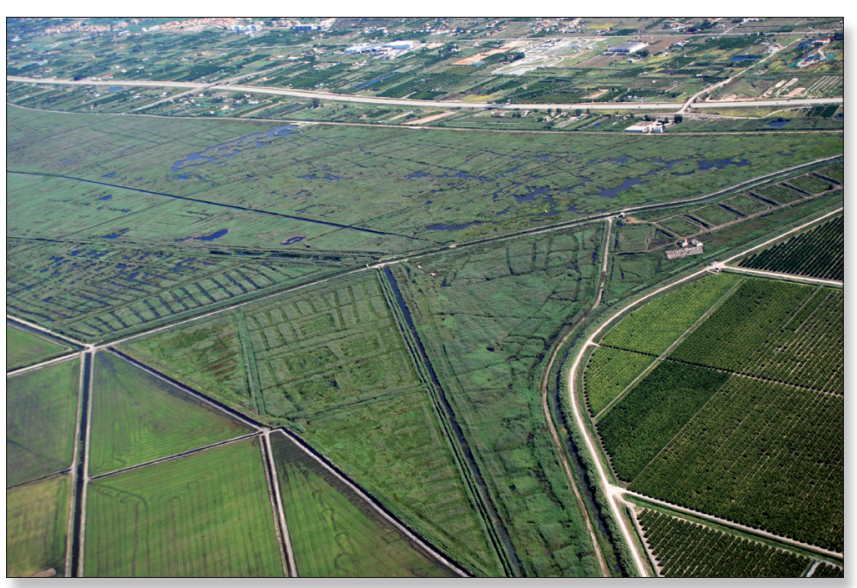

Fig. 8 - Trois faciès contrastés des anciens marjales de Pego. Cliché A. Humbert, 22/06/2008.

topographique et les photographies correspondantes. Pour l'Espagne, l'Instituto geográfico nacional fournit des cartes au $1 / 25000$ e et au $1 / 50000^{\mathrm{e}}$ qu'on retrouve sur son nouveau site d'images Iberpix (éditions anciennes et actuelles), accompagnées par les images satellitaires et photographiques aériennes et par des cartes d'occupation du sol. On peut donc suivre sur un plan les limites des unités et sous unités et connaître leur extension réelle ainsi que leur inscription dans un cadre régional plus vaste (fig. 9).

\section{I.I - La sierra littorale}

(A) présente en fait deux crêtes orientées SO-NE, selon la direction générale des chaînes subbétiques dont elle fait partie dans leur terminaison nord-orientale (caps de
San Antonio et de la Nao). La Sierra de Mustalla (au nord) et celle de Segaria (au sud) encadrent le Valle de Pego, qui n'est pas une vallée proprement dite, puisque aucune rivière ne l'occupe: il ne peut s'agir que d'une dépression tectonique, dont le plancher est occupé par un large plan incliné (glacis d'accumulation et/ou d'érosion) dont la partie inférieure a été occupée par une lagune (albufera) fermée vers la mer par un cordon littoral (arenal). Par contre plusieurs sources résurgentes apparaissent au pied des sierras calcaires de part et d'autre du Valle et donnent naissance à des riu abondants qui traversent la zone $\mathrm{B}$ et la zone $\mathrm{C}$ pour rejoindre la mer: le Bullens au nord, et le Racons au sud. Ce dernier conflue avec le riu de Molinell qui sert d'exutoire à la zone des marjales et traverse le cordon par un tracé oblique (difficulté classique de rejoindre la mer pour les graus des lagunes en empruntant les couloirs entre les cordons de dunes).

Notons que le versant nord de la sierra de Segaria a accueilli des lotissements de villas individuelles urbanizacion de "chalets perchés en "balcons 》 au dessus du Valle (Monte Pego I et II, A2) : ces opérations immobilières pour résidences secondaires ont été réalisées à partir des années 1980 dans la fièvre des constructions touristiques qui constituent la zone $\mathrm{C}$ du littoral. Il s'agit dans ce cas de la deuxième poussée de ce type de résidences : lorsque le littoral a commencé à être saturé, les promoteurs ont développé une offre de villas sur les premières pentes des sierras qui regardent la mer. Sur le territoire de la commune de Pego, qui est privée de front de mer par les communes d'Oliva au nord et de Denia au sud, seul ce type de site a pu être urbanisé par cette forme de lotissements.

\section{I.2 - La zone du piémont et de la dépression littorale}

(B) est aisément délimitée par son cadre montagneux à l'amont et sur les bords. La ville de Pego est évidemment plus visible sur les documents plans que sur l'image oblique, car la photo orthogonale montre une tache urbaine considérable, où il n'est pas difficile d'observer un centre ancien à peu près circulaire et dense, qui se distingue de quartiers d'habitat récent à l'ouest, au pied de la sierra, et de zones d'activités nouvelles à l'est, en particulier en sortie de ville le long de l'axe routier principal qui relie la ville à l'axe de circulation littorale (route nationale et autoroute). C'est un site de ville et un réseau de communication qu'on retrouve souvent le long de la côte du golfe de Valence: une petite ville centre d'une plaine agricole, transformée par l'industrialisation et l'essor du tourisme balnéaire, et reliée par une ou plusieurs pénétrantes à l'axe nord-sud.

Les différentes zones de cultures y sont maintenant visibles dans leur extension totale (à l'échelle du Valle:

- Sur le cordon littoral, la zone cultivée (B3) s'étend partout en arrière de la zone touristique, mêlant agrumes et cultures de huerta (cultures maraîchères). Les nuances agricoles décrites plus haut (« secteur de polyculture irriguée des marjales asséchés et du cordon littoral, in « légendes $\mathrm{B}$ et $\mathrm{C}$ de la figure $5 »)$. La limite de la zone touristique balnéaire montre clairement que celle-ci se développe par avancées successives au détriment de la zone cultivée se retrouvant aisément tout au long du cordon vers le nord et le sud (fig. 6 et 7). 


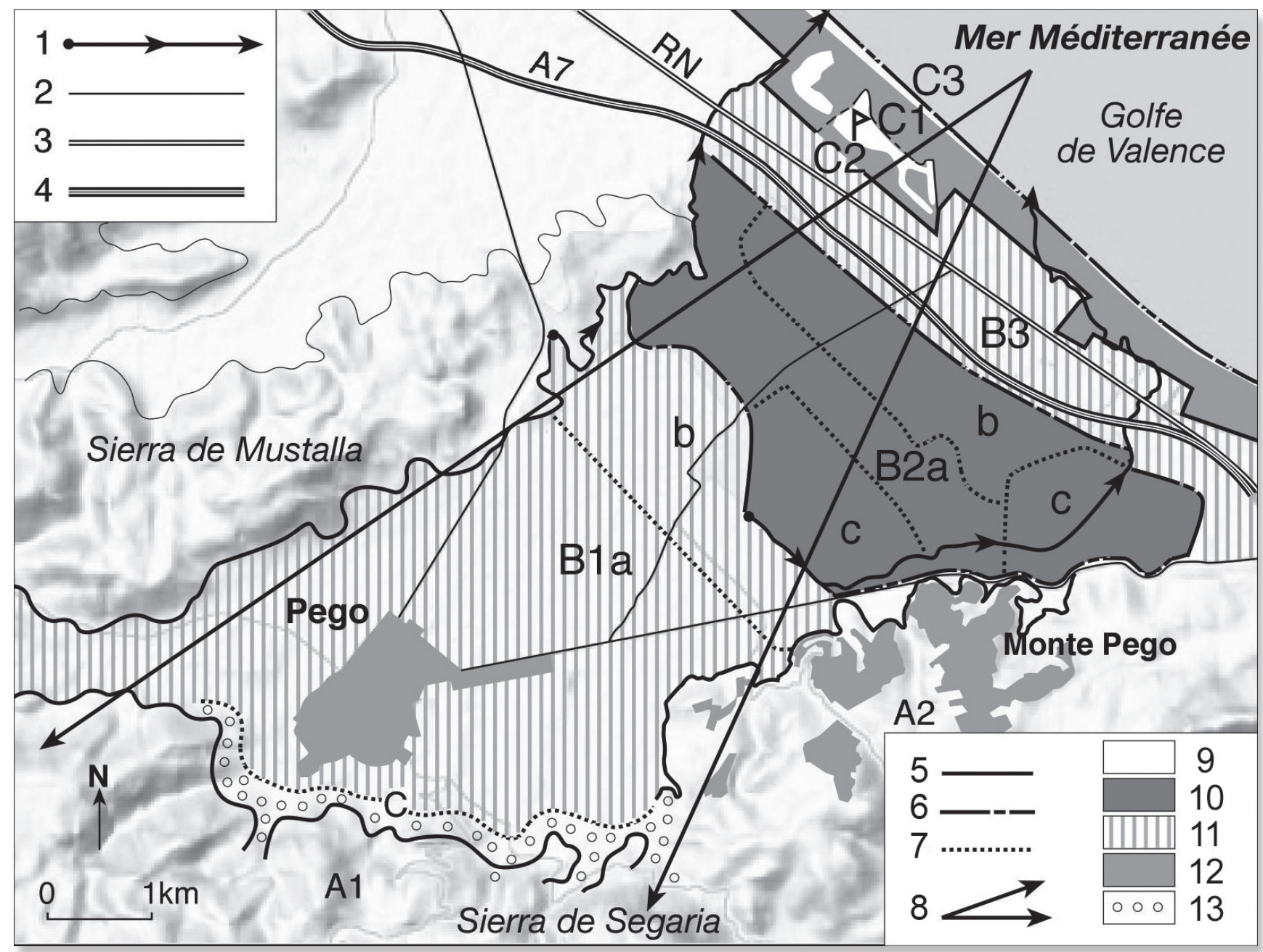

Fig. 9 - Cartographie des zones homogènes définies par l'analyse de la photographie aérienne oblique du Valle de Pego. Sources: Iberpix, Nasa worldwind geocover 2000, Google earth. 1-canal d'irrigation, 2-route secondaire, 3-route nationale, 4-autoroute, 5-limite partition niveau 1, 6-limite partition niveau 2, 7-limite partition niveau 3, 8-angle de vue de la figure 4, 9-sierra littorale, 10-rizière, 11-agrumes, 12-zones urbanisées, 13-agrumes sur terrasses anciennes.

- La zone des marjales (B2) est occupée de trois façons différentes:

- immédiatement en arrière du cordon $(\mathbf{B 2} \mathbf{b})$, des terres humides ne sont plus cultivées, alors qu'on voit par transparence les traces d'un petit parcellaire antérieur (rizières);

- plus à l'ouest une bande de rizières en grandes parcelles géométriques (B2a), dont la limite vers l'intérieur est formée par des parcelles non cultivées ou occupée par de petites surfaces légumières (Courtot, 1990);

- au sud-est un tancat (marchal d'Oriol) (B2c) : c'est un grand domaine isolé de la zone humide par une digue et un canal de drainage circulaires (tancat en valencien signifie fermé). Il est occupé par des vergers d'agrumes récents, laissant place dans l'angle oriental à un parc de loisir (zoologique) qu'on peut mettre en rapport avec la clientèle touristique du littoral.

Cette occupation différenciée s'explique par l'évolution récente de cet espace (COURTOT, 1990) : la marjal de Pego a été l'objet d'une tentative d'assainissement et de remenbrement par l'Instituto de reforma y desarollo agrario (IRYDA) en 1978-/81: les anciennes rizières en petites lanières ont été remplacées par de grandes parcelles géométriques, et une partie de l'arrozal retourne à la zone humide car les travaux de drainage se sont révélés insuffisants face au débit des sources karstiques et aux capacités des canaux de drainage vers la mer à travers le cordon. En fait les rizières n'occupent plus qu'une moitié de la zone humide, qui a été déclarée Parc naturel régional en 1994 (en exceptant le tancat d'Oriol).

*Au delà des rizières, la zone cultivée $\mathbf{B} 1$ est bien dominée par les vergers d'agrumes (huertos) même si un certain nombre de parcelles sont occupées par les cultures de huerta. On note cependant deux différenciations dans ce tissu à dominante arborée: la partie la plus basse (orientale, B1b) présente un parcellaire de lanières étroites et parallèles orientées dans le sens de la pente. Ce type de parcellaire est celui qui a servi à conquérir les marjales dans toutes les plaines littorales du golfe de Valence: un fossé de drainage long et étroit, dans le sens de la pente, fournissait la terre nécessaire pour construire sur son bord le « bancal», la bande de terre exondée qui allait être cultivée (nombreux exemples dans la huerta de Valence et dans celles de la province de Castellón). Des petites parcelles témoignent des travaux d'une petite paysannerie confrontée à l'assainissement difficile d'une zone marécageuse par de petits agriculteurs; des grands casiers sont le signe d'opérations d'aménagement par de grands propriétaires fonciers ou des organismes collectifs contemporains.

L'imagerie orthogonale permet de constater que la partie la plus haute, au contact de la sierra, dans les vallons et sur les premières pentes, est occupée par des cultures d'agrumes en terrasses, parfois dominés par des réservoirs d'eau qui sont des bassins pour l'irrigation par gravité (B1c). 
4.I.3 - Enfin la zone touristique littorale

(C) se développe sur tout le cordon du nord au sud des images de la carte et des photographies aériennes utilisées. La partie au nord du Molinell appartient à la commune d'Oliva, la partie au sud à la commune de Denia: c'est en fait un maillon d'une chaîne d'urbanisations touristiques qui couvre la majeure partie des plages du golfe de Valence (COURTOT, 2011). Chaque commune qui avait dans son territoire un morceau du bord de mer y a délivré des permis de construire, de telle sorte que chaque centre communal dans la plaine littoral a projeté son urbanización de playa . Ces dernières, en se développant de façon linéaire en front de mer ont fini par se rejoindre pour former un ruban quasi continu: l'urbanisation balnéaire est ininterrompue de Gandia à Denia.

\section{2 - La vision « horizontale » : la coupe, le profil géographique}

Celui-ci est une transposition sur une coupe topographique de l'information géographique recueillie jusqu'ici. La coupe peut être menée selon un transect précis, ou schématisée comme étant un résumé d'une situation qui concerne la région cartographiée. C'est la construction d'un inventaire inscrit dans la partition: en tant qu'inventaire géographique le long d'une coupe, cet exercice a été depuis longtemps réalisé par les géographes sur le terrain (sous forme de « cheminement ») ou sous le stéréoscope avec les couvertures photographiques aériennes existantes.

On peut maintenant tracer un profil géographique, c'est-à-dire une coupe qui intègre le relief, et les formes d'occupation humaine du sol. Le géomorphologue pourra ajouter la géologie du sol et du sous-sol, ce qui permettra de saisir les relations entre le milieu naturel (géomorphologique) et la société qui l'occupe. Dans le cas présent, le meilleur tracé de coupe doit être perpendiculaire à l'orientation générale des zones homogènes, pour fournir le maximum d'information: ce sera donc un tracé $\mathrm{SO} / \mathrm{NE}$, de la sierra littorale en arrière de Pego jusqu'à la mer (fig. 10).

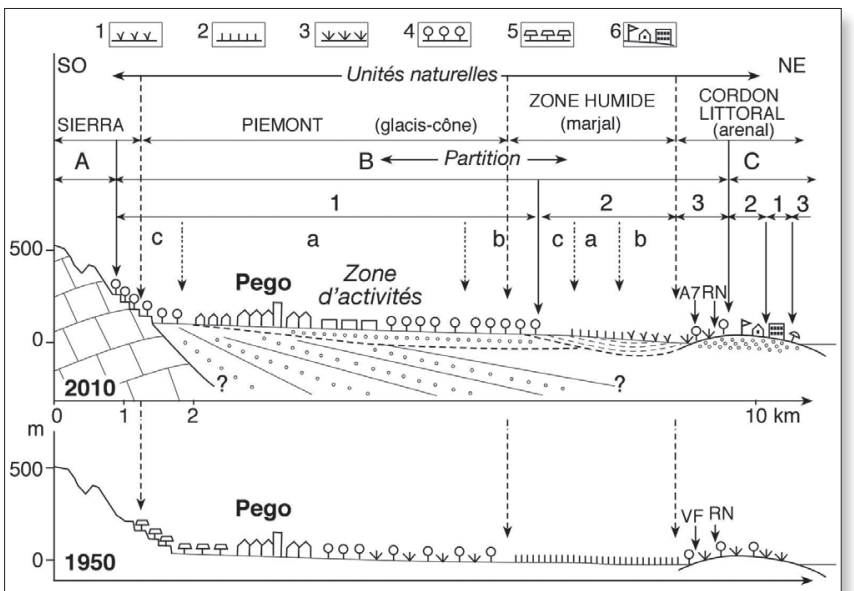

Fig. 10 - Profil schématique de l'occupation du sol dans le Valle de Pego de la sierra à la mer (2010 et 1950)

1-marais, 2-rizières, 3-cultures maraîchères, 4-agrumes, 5-oliviers, 6 -tourisme balnéaire.
Ce profil montre les rapports de proximité qu'entretiennent les différentes unités et les logiques des formes d'occupation du sol: pour les cultures, c'est une logique qui va du plus sec au plus humide, et qui dépend à la fois de l'altitude, de la nature des sols et des possibilités d'irrigation des plantes, dont le choix par la paysannerie local a dépendu des possibilités phytogéographiques et des conjonctures économiques. Le riz a occupé les marécages de la lagune au fur et à mesure de leur bonification: les agrumes et les cultures de huerta ont occupé le piémont à partir du moment où les pompages dans les nappes ont permis leur irrigation, et où le marché national et international a été ouvert et accessible à ces productions. Elles ont de la même façon occupé les sols sablonneux de l'arenal.

L'image aérienne oblique montre parfois des traces des formes d'occupation et d'organisation anciennes (antérieures) de l'espace représenté : leur figuré permet d'identifier les objets neufs, les formes en progression (constructions, défrichements, chantiers), et les formes en régression (ruines, abandons, friches...) : on peut donc enregistrer les fronts pionniers et les reculs. L'étude de ces formes anciennes, à travers les documents disponibles et les recherches de terrain permet de les reconstituer et de produire alors, en séries chronologiques, des cartes, des coupes, des blocs diagrammes, qui schématisent et résument l'évolution de l'occupation du sol parfois sur plusieurs siècles: nous nous contentons ici de revenir plus d'un demi siècle en arrière en combinant la première carte au $1 / 50000^{\mathrm{e}}$ de la région (1950) et les photographies aériennes du Vuelo americano (1956) (fig.11).

Le même profil construit à une date antérieure permet de constater les mutations qui ont affecté l'espace du Valle de Pego et modifié la partition spatiale entre les deux dates: la plus importante est celle de l'urbanisation du cordon littoral, qui a occupé la moitié d'une zone cultivée alors en huerta et agrumes, faisant disparaître en bord de mer un paysage de parcellaire cloisonné par des haies de lauriers et de cannisses caractéristique des arenales du golfe de Valence (COURTOT, 1970).

La zone humide était alors un arrozal beaucoup plus étendu et plus homogène, mais déjà handicapé par un parcellaire laniéré sous le signe de la petite paysannerie; à l'est, la marjal d'Oriol n'était pas encore « assainie ».

Enfin le piémont proprement dit était partagé en 2 zones assez nettement tranchées: l'une à l'aval déjà occupée par des cultures de huerta et des agrumes irrigués par des puits, et l'autre à l'amont occupée par des cultures sèches d'oliviers jusque sur les pentes de la sierra, où des terrasses de plus en plus étroites avec la pente avaient été construites. Les agrumes ont été le moteur de l'extension de la zone B1 : en aval au détriment des rizières (B1b), en amont au détriment des oliviers (B1c). 


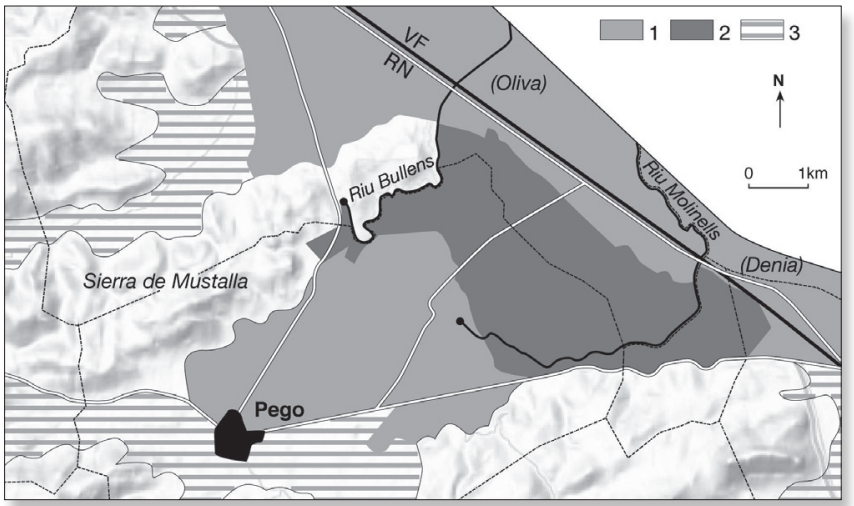

Fig. 11 - L'occupation du sol dans le Valle de Pego en 1950-1956. Sources (carte Instituto geográfico y catastral, Gandía 1/50000 $n^{\circ} 796$, $1^{\text {ère }}$ édition 1950, Vuelo americano 1956, Servicio geográfico del ejército, roll 33, $n^{\circ} 2467$ à 2469 et roll 70,5812 à 5816). 1-cultures maraîchères et agrumes, 2-rizières, 3-oliviers.

Ces profils nous introduisent ainsi à la notion importante en analyse régionale de "modélisation spatiale » et de dynamique des géosystèmes, car le schéma d'évolution que nous voyons apparaître ici est semblable à celui que nous avons décrit pour la plaine littorale de Valence (COURTOT, 1989, p. 126): les agrumes, dont la production n'a cessé de croître et de dominer l'économie valencienne de la fin du XIX ${ }^{\mathrm{e}}$ à la seconde moitié du XX $\mathrm{X}^{\mathrm{e}}$ siècle, repoussent vers les zones humides les cultures de huerta qui repoussent à leur tour les rizières. Vers les collines et les sierras bordières, les mêmes agrumes sont installés sur le piémont et les pentes à la place des cultures sèches antérieures. Avec cette nuance près, dans le cas de Pego-Oliva, que la zone humide a résisté à la conquête agricole totale par les rizières, les cultures de huertas et enfin les agrumes faute de la maîtrise complète des contraintes hydrologiques trop fortes: la transformation du tancat d'Oriol, seul espace hydrologiquement maîtrisé, des rizières aux vergers d'agrumes en est une preuve à contrario. Pour la marjal, l'écologie y a gagné, puisque la création d'un parc naturel dans les limites de cette zone humide en a fait retourner une partie au marécage. Le modèle général (celui de la plaine littorale du golfe de Valence) vérifie ainsi le modèle local (celui du Valle de Pego) et oblige à en expliquer les déformations.

\section{Conclusion}

La découverte de la vision aérienne a enthousiasmé les géographes du début du $\mathrm{XX}^{\mathrm{e}}$ siècle. Cette séduction s'est prolongée jusqu'aux années 1950 lorsque sont apparues les couvertures intégrales de photographies verticales. De nouvelles orientations de la géographie ont peu à peu détaché les chercheurs et les enseignants de la photo aérienne même si les nouvelles images spatiales en libre accès ont renouvelé l'intérêt pour ce type de document. Les images aériennes obliques n'ont pas été épargnées par le désamour qu'a connu la photographie aérienne dans son ensemble, tout au plus est-elle devenue source d'illustration; elle est pourtant un outil exceptionnel pour analyser les territoires grâce au recul qu'elle permet et à la réalité des paysages qu'elle conserve. Ces images qui se dérobent à toute normalité géométrique peuvent néanmoins servir, avec toute l'information qu'elles contiennent, à restituer des représentations "redressées » et mises à l'échelle, des géosystèmes qu'elles révèlent. Outil de chercheur, la photographie aérienne oblique est aussi un incomparable support didactique pour la formation des étudiants à l'analyse de l'espace.

\section{Bibliographie}

AMAT Jean-Paul \& GRATAloup Christian, (1992), «Interface, Du paysage au système » in AMAT J.-P. \& RoBIC M.-C., Géographiques-Espace à vivre/Espace vécu, CNDP, coll. Autrement dit-niveau lycée.

AMAT Jean-Paul, (1988), "La photographie militaire aérienne, source pour l'étude des paysages ruraux de la Grande Guerre » in 14-18-Vues d'en haut, La photographie aérienne pendant la guerre de 1914-1918, musée de l'Armée, musée d'Histoire contemporaine-BDIC, Paris, p. 37-42.

BAzZAna André et Humbert André, (1983), Prospections aériennes. Les paysages et leur histoire, Publications de la Casa de Velázquez, Série Recherches en sciences sociales, VII, Madrid, 200 p., 57 photographies aériennes obliques en couleur.

BIElZA DE ORY, Vicente, HumBerT André et GARCíA RUIZ José-María, (1994), Geografía de los paisajes de Aragón, Diputación General de Aragón, Zaragoza, 150 p., 130 photographies aériennes obliques en couleur.

COLSON Hervé, (2010), La Lorraine vue du ciel, Un regard inédit sur le patrimoine majeur, Place Stanislas, 350 photos.

COURTOT Roland, (1969), «La photographie aérienne et les paysages agraires dans la basse vallée du Jucar (province de Valencia, Espagne) », Actes du 94 congrès national des sociétés savantes, section géographie, Pau, Paris, BN 1970, p. 67-80.

—, (1989), Campagnes et villes dans les huertas valenciennes, mémoires et documents de géographie, CNRS, Paris, 191 p.

—, (1990), "Le littoral valencien. Des huertas traditionnelles au système urbain ", p. 15-31 in F. FOURNEAU, A. HUMBERT et M. VAlenZUela (coord.), Géographie d'une Espagne en mutations, (Prospections aériennes II), Publications de la Casa de Velázquez, Série Recherches en Sciences Sociales, IX, Madrid, 261 p.

—, (2011), «Littoralisation et nouveaux modèles spatiaux dans la communauté du Pays valencien » in A. HUMBERT, F. MOLINERO et M. VALENZUELA (coord.), España en la Unión europea, Casa de Velázquez, Madrid, p. 107-124.

DefFontaines Pierre et Jean-Brunhes Delamarre Mariel, (1955-1964), Atlas aérien. France, Gallimard, 5 tomes, Paris.

DEMANGEOn Albert, (1946), La France économique et humaine, Géographie Universelle, tome VI, Armand Colin, Paris, 460 p.

DUMARCHE Lionel, (1988) «La photographie aérienne 1914-1918: une nouvelle arme de guerre » in 14-18-Vues d'en haut,

La photographie aérienne pendant la guerre de 1914-1918, musée de l'Armée, musée d'Histoire contemporaine - BDIC, Paris, p. 8-15. 
FLÜCKIGER O., (1924), Die Schweiz aus des Vogelschau, Zürich, 243 vues aériennes presqu'exclusivement obliques.

FONTAVElLA GONZALEZ V., (1952), La huerta de Gandia, Inst. Juan Sabastian Elcano, CSIC, Zaragoza, 404 p.

Fourneau Francis, Humbert André et VAlenzuela Manuel, (1990), Géographie d'une Espagne en mutation. Prospections aériennes II, Publications de la Casa de Velázquez, Série recherches en sciences sociales, IX, Madrid, 269 p., 110 photographies aériennes obliques en couleur.

HUMBERT André, (2001), «The areal Field » in Dydia DE LYSER et Paul F. STARRS (ed.), Doing Fieldwork, Geographical Review, vol. $91, \mathrm{n}^{\circ} 1$ et 2 , p. 273-284

—, (2012), Le géographe et le tapis volant, Publications de la Casa de Velázquez, Essais de la Casa de Velázquez, vol. 5, Madrid, 190 p. HUMBERT André et RENARD Colette, (2008), « Observer et représenter les paysages » in Paul ARNOULD et Guy BAUdELLE, (coord.), Construire les territoires, Historiens Géographes, nº 403, p. 69-79.

Humbert André, Molinero Hernando Fernando et VAlenzuela Rubio Manuel (ed.), (2011), España en la Unión Europea. Un cuarto de siglo de mutaciones territoriales, Publications de la Casa de Velázquez, Collection de la Casa de Velázquez, vol. 121, Madrid, 280 p., 112 photographies aériennes obliques en couleur.

LEE Willis T., The face of the Earth as seen from the air, Amer. Geogr. Soc. Spec. Publication, no 4, New York, 1922, surtout des photographies aériennes verticales.

LEGOUY François, (2006), Le paysage des Hautes-côtes de Beaune: de la vision du «dedans » à la vision du « dessus » in Sud-Ouest européen, $\mathrm{n}^{\circ} 21$, p. 9-18.

LIZET Bernadette et RAVIGNAN François (de), 1987, Comprendre un paysage-Guide pratique de recherche, INRA, coll. Écologie et aménagement rural, Paris, $148 \mathrm{p}$.

MACLEAN Alex, (2008), Over, Visions aériennes de l'American Way of Life: une absurdité écologique, Dominique Carré Éditeur-La Découverte, 337 p.

MARTONNE Emmanuel (de), (1924), La photographie d'avion au service de la géographie en Suisse et aux États-Unis, Annales de Géographie, t. 33, n 185, p. 484-488, en ligne [http://www.persee.fr/web/revues/home/prescript/article/ geo_0003-4010_1924_num_33_185_9439].

MARTONNE Emmanuel (de), (1948), Géographie aérienne, Albin Michel, coll. Sciences d'aujourd'hui, Paris, 241 p.

MOLINERO Fernando, OJEDA Juan Francisco et TORT Joan (coord.), (2011), Los paisajes agrarios de España, caracterización, evolución y tipificación, Publicaciones del Ministerio de Medio Ambiente y Medio Rural y Marino, Madrid, 606 p., nombreuses photographies aériennes obliques en couleur.

PinChemel Philippe et Pinchemel Geneviève, (1987), « Lire les paysages », La Documentation photographique, $\mathrm{n}^{\circ} 6088$.

ROSNAY Joël (de), (1975), Le macroscope. Vers une vision globale, Le Seuil, coll. Points, Paris, 313 p. 
